\title{
Backward Induction in Games without Perfect Recall
}

\author{
John Hillas and Dmitriy Kvasov
}

CMSS Workshop, February 2012 


\section{Backward Induction}

The game theorists who defined the equilibrium concepts that we now think of as various forms of backwards induction, namely subgame perfect equilibrium (Selten, 1965), perfect equilibrium (Selten, 1975), sequential equilibrium (Kreps and Wilson, 1982), and quasi-perfect equilibrium (van Damme, 1984), explicitly restricted their analysis to games with perfect recall. In spite of this the concepts are well defined, exactly as they defined them, even in games without perfect recall. There is now a small literature examining the behaviour of these concepts in games without perfect recall. Jeff Kline (2005) and Joe Halpern and Rafael Pass (2008) look at what happens in games without perfect recall to solutions defined in exactly the same way as they were defined in games with perfect recall. 


\section{Backward Induction without Perfect Recall}

We shall argue that in games without perfect recall the original definitions are inappropriate. Our reading of the original papers is not that the authors were unaware that their definitions did not require the assumption of perfect recall, but rather that they were aware that without the assumption of perfect recall the definitions they gave were not the "correct" ones. In this paper we give definitions of two of these concepts, sequential equilibrium and quasi-perfect equilibrium that identify the same equilibria in games with perfect recall and behave well in games without perfect recall. 


\section{The Nature of Our Project}

What we want to do is to give definitions of the "backward induction" solutions-in this paper sequential equilibrium and quasi-perfect equilibrium-that

1. "coincides" with the original definitions in games with perfect recall, and 


\section{The Nature of Our Project}

What we want to do is to give definitions of the "backward induction" solutions-in this paper sequential equilibrium and quasi-perfect equilibrium - that

1. "coincides" with the original definitions in games with perfect recall, and

2. satisfies the same properties in games without perfect recall as it does in games with perfect recall. In particular the inclusions that a proper equilibrium is a quasi-perfect equilibrium and a quasi-perfect equilibrium is a sequential equilibrium. 


\section{Informal Definitions}

\section{Definition}

A player has perfect recall if, at each of his information sets he remembers what he knew and what he did in the past. This concept was originally defined by Kuhn (1953). Later an equivalent definition was given by Selten (1975).

In games without perfect recall we distinguish between linear games (the games defined by Kuhn) and nonlinear games (an extension by Isbell (1957) and more recently discussed under the name "absent-mindedness" by Piccione and Rubinstein (1997)). In linear games each play of the game reaches an information set at most once. For the moment we shall be restricting attention to linear games. The issues are not so different in nonlinear games and, time permitting, we shall return to discuss an extension of the concepts to nonlinear games at the end of this talk. 


\section{Informal Definitions (cont)}

\section{Definition}

A pure strategy in an extensive form game for Player $n$ is a function that maps his information sets to the set of actions such that each information set is mapped to an action available at that information set.

\section{Definition}

A behaviour strategy in an extensive form game for Player $n$ is a function that maps his information sets to the set of probability distributions over the set of actions such that each information set is mapped to a probability distribution that puts all weight on actions available at that information set.

\section{Definition}

A mixed strategy in an extensive form game for Player $n$ is a probability distribution over the player's pure strategies. 


\section{Pure Strategies, Behaviour Strategies, and Mixed Strategies}

An immediate implication of these definitions is that the set of pure strategies is embedded in both the set of behaviour strategies and the set of mixed strategies. A pure strategy $s$ is equivalent to the behaviour strategy that takes each information set to the probability distribution that puts weight 1 on the action that $s$ selects at that information set. And $s$ is equivalent to the mixed strategy that puts weight 1 on $s$. 


\section{Kuhn's Theorem}

Kuhn (1953) showed that if a player has perfect recall then each of his mixed strategies has an equivalent behaviour strategy, that is, a behaviour strategy that will induce the same distribution on terminal nodes as the mixed strategy, whatever the other players may choose. If the player does not have perfect recall this will not be true for all mixed strategies. In a linear game each behaviour strategy of a player has an equivalent mixed strategy. 


\section{Nonexistence of Equilibria in Behaviour Strategies}

An immediate implication of the definitions of strategies is that the set of pure strategies is embedded in both the set of behaviour strategies and the set of mixed strategies. A pure strategy $s$ is equivalent to the behaviour strategy that takes each information set to the probability distribution that puts weight 1 on the action that $s$ selects at that information set. And $s$ is equivalent to the mixed strategy that puts weight 1 on $s$.

This means that in games in which there is a unique equilibrium in mixed strategies and that mixed strategy profile is not equivalent to a profile of behaviour strategies there is no equilibrium in behaviour strategies. For, if there were, then the mixed strategy profile equivalent to that profile would also be an equilibrium in mixed strategies. 
We first consider a game without perfect recall given in the next slide. (This is a very slight modification of a game considered by Kuhn (1953).) In this game there is a unique equilibrium in mixed strategies. For one of the players the equilibrium mixed strategy is not equivalent to any behaviour strategy. 
Example 1

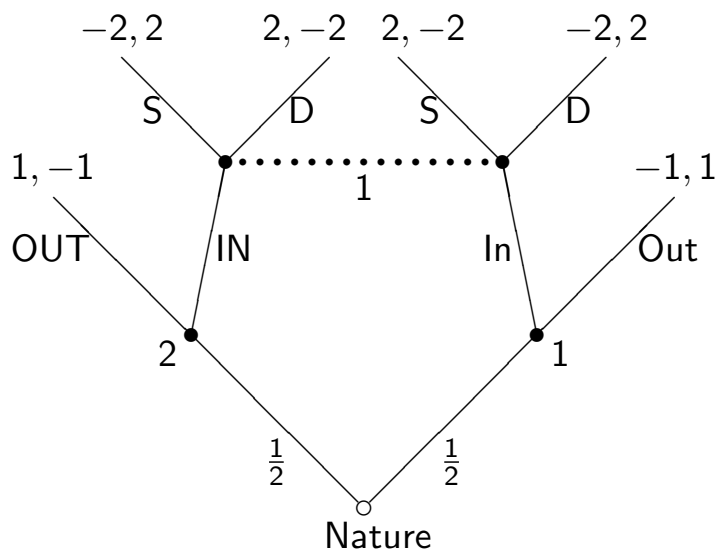

Figure: A game without perfect recall. 


\section{Example 1: Normal Form}

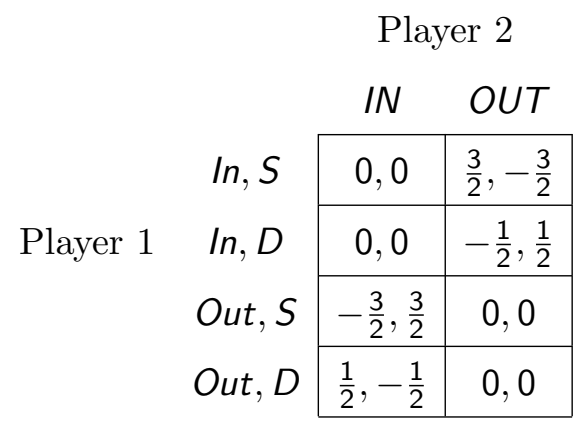

Figure: The normal form game corresponding to the extensive form game above 
Example 2

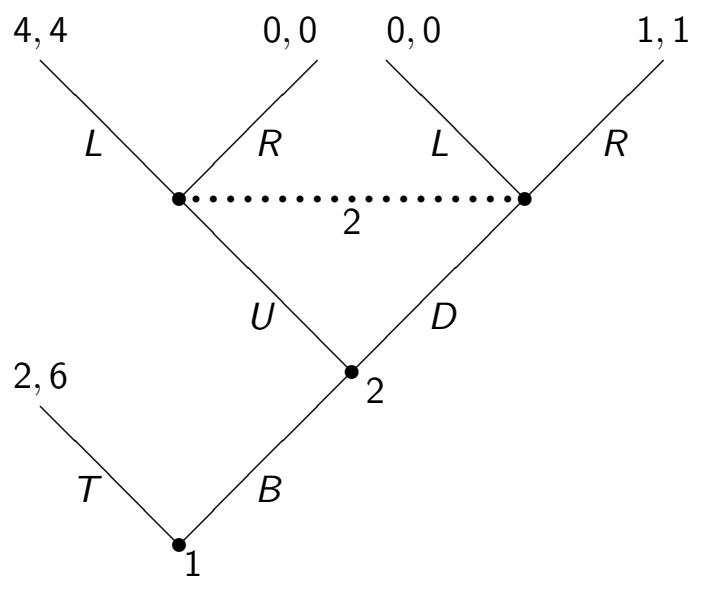

Figure: No One Deviation Principle 


\section{Example 3}

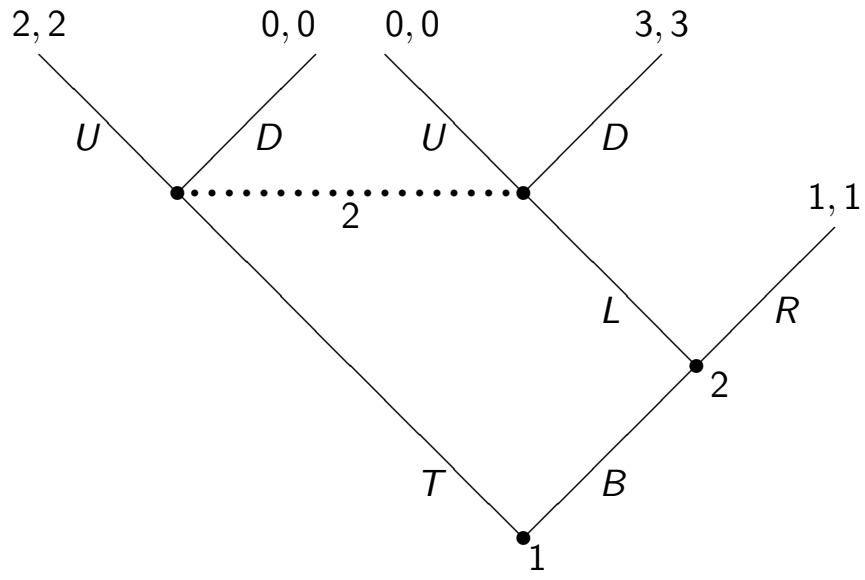

Figure: Cannot deviate at all information sets 
We shall now define sequential equilibria and quasi-perfect equilibria. Since we have seen that we cannot hope to satisfy a one-deviation property and that it will be necessary to consider players deviating simultaneously at a number of information sets we shall define beliefs not at an information set but at a collection of information sets. In the original definition of sequential equilibrium beliefs were defined as a probability distribution over the nodes of an information set. Here we define beliefs as distributions over the pure strategies that are being played, including Nature's "strategy." First we need a bit of notation. 


\section{Notation}

We shall denote the set of players by $N$, the set of Player $n$ 's pure strategies by $S_{n}$, the set of Player $n$ 's behaviour strategies by $B_{n}$ with $B=\times_{n \in N} B_{n}$, and the set of Player n's mixed strategies by $\Sigma_{n}$ with $\Sigma=\times_{n \in N} \Sigma_{n}$. We shall let $S=\times_{n \in\{0\} \cup N} S_{n}$, that is, when we refer to profiles of pure strategies we shall specify also the "strategy" of Nature.

We shall denote the collection of information sets by $\mathcal{H}$, with $\mathcal{H}_{n}$ the information sets of Player $n$. We shall also consider the collection of non-empty subsets of $\mathcal{H}_{n}$ which we shall denote $\overline{\mathcal{H}}_{n}$. An element of $\overline{\mathcal{H}}_{n}$ is a collection of information sets of Player $n$. 


\section{System of Beliefs}

\section{Definition}

A system of beliefs $\mu$ defines, for each $n$ in $N$ and each $H$ in $\overline{\mathcal{H}}_{n}$ a distribution $\mu\left(s_{0}, s_{1}, \ldots, s_{N} \mid H\right)$ over the profiles of pure strategies that reach $H$. Given $\mu$ we also consider $\mu_{S_{n}}\left(s_{n} \mid H\right)$ and $\mu_{S_{-n}}\left(s_{-n} \mid s_{n}, H\right)$ the marginal distribution on $S_{n}$ given $H$ and the conditional distribution on $S_{-n}$ conditional on $S_{n}$ and $H$.

Recall that we have seen above that a player's beliefs at an information set about what strategies the other players are playing may differ depending on what pure strategy he himself is playing. Notice also that we include the (pure) strategy of Nature in the list of strategies over which Player $n$ has beliefs. 


\section{Sequential Equilibrium}

We first define sequential equilibria.

Definition

Given a pair $(\sigma, \mu)$ we say that the pair is consistent (or is a consistent assessment) if there is a sequence of completely mixed strategy profiles $\sigma^{t} \rightarrow \sigma$ with $\mu^{t}$ a system of beliefs obtained from $\mu^{t}$ as conditional probabilities and $\mu^{t} \rightarrow \mu$.

\section{Definition}

Given a pair $(\sigma, \mu)$ we say that the pair is sequentially rational if, for each $n$, for each $H$ in $\overline{\mathcal{H}}_{n}$, and for each $s_{n}$ in $S_{n}$ if $\mu_{S_{n}}\left(s_{n} \mid H\right)>0$ then $s_{n}$ maximises

$$
E_{\mu_{S_{-n}}\left(s_{-n} \mid s_{n}, H\right)} u_{n}\left(t_{n}, s_{-n}\right)
$$

over the set of all $t_{n}$ in $S_{n}$ such that $t_{n}$ differs from $s_{n}$ only at information sets in $H$. 


\section{Sequential Equilibrium}

\section{Definition}

Given a pair $(\sigma, \mu)$ we say that the pair is a sequential equilibrium if it is both consistent and sequentially rational. 


\section{Quasi-perfect Equilibrium}

Quasi-perfect equilibria are defined in a similar way.

Definition

A strategy profile $\sigma$ is a quasi-perfect equilibrium if there is a sequence of completely mixed strategy profiles $\sigma^{t} \rightarrow \sigma$ with $\mu^{t}$ a system of beliefs obtained from $\mu^{t}$ as conditional probabilities and $\mu^{t} \rightarrow \mu$ and for each $n$, for each $H$ in $\overline{\mathcal{H}}_{n}$, and for each $s_{n}$ in $S_{n}$ if $\mu_{S_{n}}\left(s_{n} \mid H\right)>0$ then $s_{n}$ maximises

$$
E_{\mu_{S_{-n}}^{t}\left(s_{-n} \mid s_{n}, H\right)} u_{n}\left(t_{n}, s_{-n}\right)
$$

over the set of all $t_{n}$ in $S_{n}$ such that $t_{n}$ differs from $s_{n}$ only at information sets in $H$.

Observe that the definitions of sequential equilibrium and quasi-perfect equilibrium differ only in use of $\mu^{t}$ rather than $\mu$ in defining the expected utility that is maximised. 


\section{Results}

The first two results say that in games with perfect recall we obtain the "same" equilibria as the original definitions.

\section{Proposition}

If the game has perfect recall then if $(\sigma, \mu)$ is a sequential equilibrium then there is a behaviour strategy profile $b$, equivalent to $\sigma$ in the sense of Kuhn, that is the strategy part of a sequential equilibrium according to the definition of Kreps and Wilson (1982). Moreover for any sequential equilibrium in the sense of Kreps and Wilson there is an equivalent mixed strategy $\sigma$ and a system of beliefs $\mu$ such that $(\sigma, \mu)$ is a sequential equilibrium.

\section{Proposition}

If the game has perfect recall then if $\sigma$ is a quasi-perfect equilibrium then there is a behaviour strategy profile $b$, equivalent to $\sigma$ in the sense of Kuhn, that is a quasi-perfect equilibrium according to the definition of van Damme (1983). Moreover, for any quasi-perfect equilibrium in the sense of van Damme there is an equivalent mixed strategy profile that is a quasi perfect 


\section{More Results}

The next two results say that the relation between the concepts is as it was in games with perfect recall.

Proposition

Every quasi-perfect equilibrium is a sequential equilibrium.

Proposition

For a generic class of extensive form games every sequential equilibrium is a quasi-perfect equilibrium. 


\section{Another Result}

Finally we have the result proved for games with perfect recall by van Damme (1983) and Kohlberg and Mertens (1986) relating quasi-perfect and sequential equilibria to proper equilibria (Myerson 1978) of the normal form.

\section{Proposition}

Every proper equilibrium is a quasi-perfect equilibrium (and hence a sequential equilibrium).

Since every game has a proper equilibrium this result also implies the existence of sequential and quasi-perfect equilibria. 